\title{
TOMOGRAPHY OF CANISTERS FOR SPENT NUCLEAR FUEL
}

M. Österlund ${ }^{* 1}$, J. Blomgren ${ }^{1}$, J. Donnard ${ }^{1,4}$, A. Flodin ${ }^{1}$, J. Gustafsson ${ }^{1}$, M. Hayashi $^{1,5}$, P. Mermod ${ }^{1}$, L. Nilsson ${ }^{1}$, S. Pomp ${ }^{1}$, L. Wallin ${ }^{1}$, A. Öhrn ${ }^{1}$, A.V. Prokofiev ${ }^{2}$

${ }^{1}$ Department of Neutron Research, Uppsala University, Sweden

${ }^{2}$ The Svedberg Laboratory, Uppsala University, Sweden

${ }^{3}$ Fast Neutron Research Facility, Chiang Mai University, Thailand

${ }^{4}$ Ecole des Mines de Nantes, France

${ }^{5}$ Department of Advanced Energy Engineering Science, Kyushu University, Japan

E-mail: Michael.Osterlundatsl.uu.se

\begin{abstract}
Sweden and Finland are preparing for final deposition of spent nuclear power fuel. The adopted method is to encapsulate spent nuclear fuel in copper canisters filled with iron before deposition in a deep bedrock repository. The canisters will have a diameter of about one metre, which makes examination of the content in sealed canisters virtually impossible with any known technique today.

Two methods for tomography of sealed canisters have been studied, high-energy neutron tomography and cosmic-ray muon tomography. Monte Carlo simulations using MCNPX have shown that it would indeed be possible to produce images of good resolution of thick massive objects, like these canisters, using high-energy neutrons. The cost for installing such a method would, however, be very high. GEANT simulations, supported by experimental tests, indicate that tomography using the natural flux of cosmic-ray muons results in images of lower quality, but to a much more modest cost, acceptable to the application.
\end{abstract}

International Workshop on Fast Neutron Detectors and Applications

April, 3 - 6, 2006

University of Cape Town, South Africa

${ }^{*}$ Speaker. 


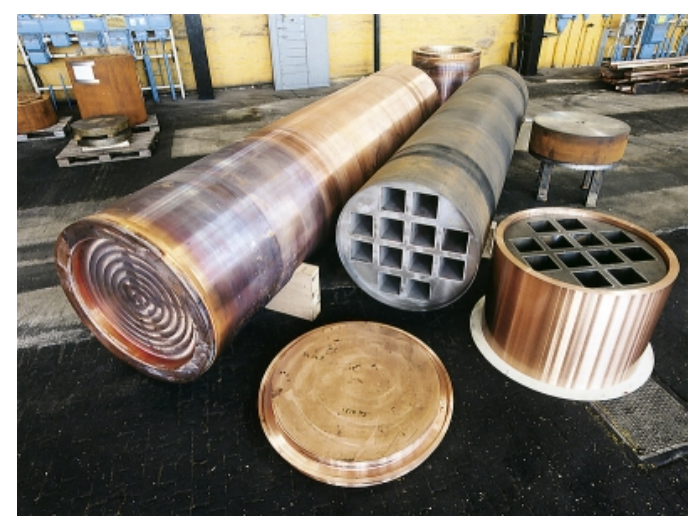

Figure 1: Canisters for spent BWR nuclear fuel. The canister consists of a cylindrical copper shell with a pressure-bearing insert of nodular iron. The outer diameter is $1.05 \mathrm{~m}$ and the length $4.83 \mathrm{~m} \mathrm{[1]}$.

\section{Introduction}

In Swedish nuclear power plants, a once-through fuel cycle is adopted where it is envisioned that the spent nuclear fuel will be encapsulated in copper canisters with iron inserts as seen in Fig. 1. The canisters will be deposited in bedrock, embedded in clay, at a depth of about $500 \mathrm{~m}$ [1]. The rock is expected to isolate and protect the waste and also to provide a stable chemical environment that will not change for the next 100000 years.

Today, the only known method for investigating the interior of a sealed canister is to open it, which is not a viable proposition. Consequently, verification of the content of canisters would have to be based on documentation and seals, which would have limited reliability. In order to adress this issue, two possible methods for radiograpich imaging of thick massive objects have been investigated, fast-neutron radiography and cosmic-muon radiography.

Research on high-energy $\left(E_{\mathrm{n}}>15 \mathrm{MeV}\right)$ neutron radiography/tomography has been performed at Los Alamos National Laboratory LANL since 1990 [2]. Unfortunately, no results have been published since 1997 when the project was classified. Because of the high cost associated with the production and the detection of high-energy neutrons, alternative lower cost methods are of particular interest. One such method, cosmic-ray muon radiography [3], that is being developed for the detection of nuclear contraband by LANL may be of used for inspection of canisters for spent nuclear fuel.

\section{Neutron tomography}

High-energy neutron tomography offers unique possibilities compared to other imaging techniqies, most notable great penetrating power and sensitivity to light elements. In general, the penetration of neutrons increases with energy, opening up the possibility for imaging of large massive objects, which is impossible with the tomographic methods commonly available today. For many nuclides the total cross section, $\sigma_{T}$, exhibits a fairly wide minimum around $300 \mathrm{MeV}$ [4] resulting in maximum penetration. High energy neutrons are usually produced through direct particleneutron interactions of charged particles against light nuclei or by spallation. This involves the use 
of accelerators such as synchrotrons or linacs, the cost of which is a steep function of energy. Also, high energy neutrons are difficult to detect, often involving a conversion process followed by the detection of the resulting charged particles in position sensitive detectors such as drift-chambers. Considering the economical and technical difficulties of generating high-energy neutrons and the availablitity of existing facilities, $100 \mathrm{MeV}$ has been found to be a reasonable good compromise for the present application.

\section{Simulations of neutron radiography in MCNPX}

In order to investigate the potential of fast-neutron imaging simulations have been performed in MCNPX [5]. The studies have been concentrated on general aspects of fast-neutron tomography, i.e., contrast, the effect of voids inside dense objects and element sensitivity [6].

For the simulations, a simple axial-symmetric geometry consisting of a $100 \mathrm{MeV}$ point neutron source, an object in the shape of a truncated right angle cone and an ideal detector, was used. The center of the $1.0 \mathrm{~m}$ thick target was situated $4.5 \mathrm{~m}$ from the source and the detector was positioned $5.5 \mathrm{~m}$ from the source.

The contrast between a massive object and air was studied using a solid iron object. The observed transmission of neutrons was approx. $2 \cdot 10^{-5}$. When observing the detector signal near the edge of the shadow projected on the detector by the object, it was observed that the signal falls off by a factor $10^{-5}$ over a distance of less then $1 \mathrm{~mm}$, which is indicative of the resolution.

In order to simulate the effects of voids, a composite iron object consisting of a hollow truncated cone with a wall thickness of $1.0 \mathrm{~cm}$, into which is inserted another solid truncated cone such as to create a uniform $1.0 \mathrm{~mm}$ wide void between the cones was used. Simulations were performed with the void extending either along the whole length of the object or being confined to the interior of the object. In the latter case, voids of length $20 \mathrm{~cm}, 30 \mathrm{~cm}$ and $60 \mathrm{~cm}$ were simulated.

The viods gave rise to detector signals that in 1D projections are well described by Gaussians. Voids with lengths down to $30 \mathrm{~cm}$ could be clearly seen, but for a $20 \mathrm{~cm}$ long void the signal was lost in the statistical fluctuations. In all cases the width of the Gaussian was approximately 0.12 $\mathrm{cm}$, implying an intrinsic resolution of $\sigma=0.04 \mathrm{~cm}$.

The attenuation of neutrons travelling through any material is caused by nuclear reactions and elastic scattering. Limits on the attenuation can be derived using the total cross section and the reaction cross section, the former giving the maximum limit and the latter giving the minimum limit.

In order to investigate the possibility of distinguishing between different materials, simulations with solid objects made of $\mathrm{Fe}, \mathrm{U}, \mathrm{Pb}$ and liquid $\mathrm{H}_{2}$ were performed. The transmissions through $\mathrm{Fe}$ and $\mathrm{U}$ are approximately equal, $2 \cdot 10^{-5}$, whereas for $\mathrm{Pb}$ it is $4 \cdot 10^{-5}$. As a comparison, the observed transmission for liquid $\mathrm{H}_{2}$ was 0.80 .

As could be expected the attenuation in liquid $\mathrm{H}_{2}$, which has a low density and a small total cross section, deviates significantly from the attenuation in the high- $Z$ elements. The only nonelastic process is capture, ${ }^{1} \mathrm{H}(\mathrm{n}, \gamma)^{2} \mathrm{H}$, which has a very small cross section. This means that in liquid $\mathrm{H}_{2}$ almost all attenuation is due to elastic scattering and because of the fairly isotropic angular distribution of elastic scattering against hydrogen, almost all elastically scattered neutrons are lost. 
For the high- $\mathrm{Z}$ elements $\mathrm{Fe}, \mathrm{Pb}$ and $\mathrm{U}$ the elastic scattering angular distribution is strongly forward-peaked and a large fraction of the elastically scattered neutrons are detected close to the position of the unscattered neutrons. The attenuation in $\mathrm{Pb}$ is a factor of two less than what is the case in $\mathrm{Fe}$ and $\mathrm{U}$. This effect is expected because the number of nuclei per volume in $\mathrm{Pb}$ is fairly small compared to $\mathrm{Fe}$ and $\mathrm{U}$. The simulated attenuations in $\mathrm{Fe}$ and $\mathrm{U}$ are similar even though the cross section for Fe is much smaller than for $\mathrm{U}$. On the other hand, the number of nuclei per unit volume is larger for Fe. It is possible that these two effects happen to balance each other at this specific energy, resulting in a similar attentuations. The effects of small-angle scattering, which is different for Fe and U, can influence the result. Small-angle scattering can also be expected to change with energy unequally for these two elements. It is therefore conceivable that a combination of neutron tomography at more than one energy could result in element sensitivity. Quantitative assessment of this hypothesis would require repetition of the simulations above at higher energies.

\section{Cosmic-ray muon tomography}

High-energy muons are created when cosmic rays interact with nuclei in the upper layers of the atmosphere. At the surface of the earth the average energy is $E_{\mu}=3-4 \mathrm{GeV}$, the flux is approximately $10^{4} \mathrm{~m}^{-2} \mathrm{~min}^{-1}$ and the angular distribution with respect to zenith is close to $\cos ^{2}(\theta)$. In contrast to neutrons which only interacts strongly, muons interact through Coulomb and weak forces. At energies typical for cosmic-ray muons, the stopping power is approx. $2.2 \mathrm{MeVcm}^{2} \mathrm{~g}^{-1}$ giving the muons a range of approx. $1.7 \mathrm{~m}$ in Fe and $0.7 \mathrm{~m}$ in $\mathrm{U}$ [12] which is sufficient for the intended application.

Because of the low flux of muons, the measurement time necessary to obtain sufficient statistics for conventional attenuation measurements [10] is prohibitive. An alternative approach is needed where as much information about an object as possible is inferred from each individual muon. A practical proposition is to measure the scattering that muons are subjected to when traversing an object.

The scattering distribution of muons is approximately Gaussian [11]

$$
\frac{d N}{d \theta}=\frac{1}{\sqrt{2 \pi} \theta_{0}} e^{-\frac{\theta^{2}}{2 \theta_{0}^{2}}} .
$$

and the width of the distribution depends on material and muon properties

$$
\theta_{0}=\frac{13.6 \mathrm{MeV}}{\beta c p} \sqrt{\frac{L}{L_{0}}}\left[1+0.038 \ln \left(\frac{L}{L_{0}}\right)\right],
$$

where $p$ is the momentum of the particle in $\mathrm{MeV} / \mathrm{c}, L_{0}$ is the radiation length and $L$ is the thickness of the target. Generally speaking, the radiation length decreases with the charge density of the material. A high- $Z$ material with high density has a shorter radiation length and therefore muons will be deflected by a larger angle in a high- $Z$ material. For $\mathrm{Fe}, \mathrm{Pb}$ and $\mathrm{U}$ the mean scattering angles are $10.8 \mathrm{mrad}, 20.0 \mathrm{mrad}$ and $26.9 \mathrm{mrad}$ respectively. For low- $Z$ materials the average scattering angle is too small to be of any particular use for radiography purposes.

In order to investigate the possibilities of this method a small-scale experiment system has been developed for initial experiments using available equipment $[7,8]$ and simulations of muon scattering in canisters for spent nuclear fuel have been performed [9]. 


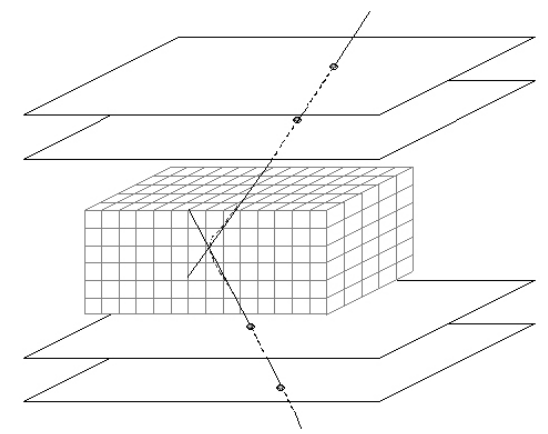

Figure 2: Schematic of a detector system. Two position sensitive detectors above the target area and two below provide muon tracking information. The actual track of the muon through the target is approximated by two straight lines in order to determine a virtual scattering point. The target volume is divided into voxels, i.e., $3 \mathrm{D}$ pixels.

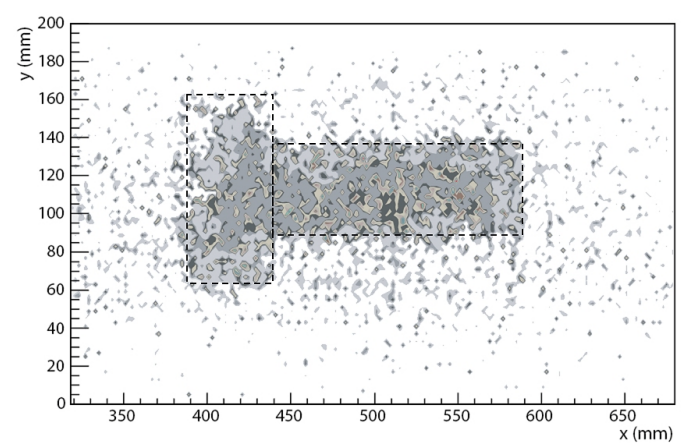

Figure 3: A 2D image of a $50 \mathrm{~mm}$ thich T-shaped target reconstructed from approx. 3500 POCAs. The dashed lines indicate the actual outline of the target.

\subsection{Experimental measurements}

For the small-scale experiment, pairs of drift chambers positioned above and below a target area were used for muon tracking. Plastic scintillators placed at the extreme top and bottom of the setup were used for particle identification and triggering 2. A simple object of lead in the shape of the character T served as target.

Because muons are continuously scattered by the electric charge of atoms in the target material, the actual path of individual muons inside the target volume cannot be determined and assumptions have to be made. One simple assumption is to consider the continous scattering to be taking place in a single point. This virtual scattering point is simply determined by extrapolating entrance and exit tracks reconstructed from drift chamber information and determining the point in space that is closest to both tracks. This point of closest approach (POCA) is considered a good point estimate for where the muon is scattered.

In order to perform the image reconstruction a target volume enclosing the target is divided into voxels, i.e. 3D pixels. For each POCA determined, the corresponding voxel is incremented using the scattering angle squared as a weighting function. The weighting function is motivated 

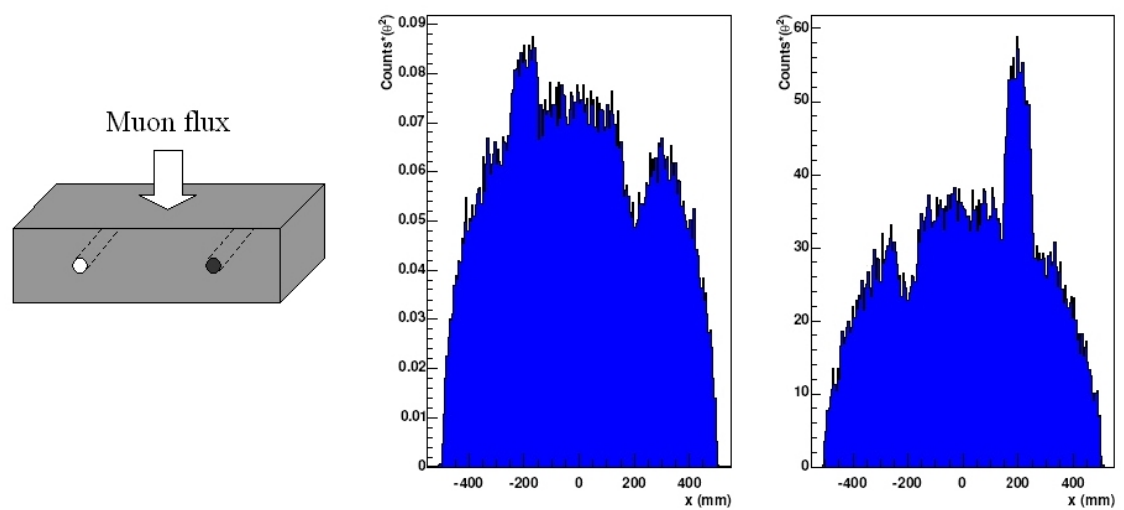

Figure 4: 1D-projections of the image obtained from a rectangular slab of iron $l \times d \times h=1000 \times 200 \times$ $250 \mathrm{~mm}^{3}$. In the slab there are two cylindrical voids $(d=50 \mathrm{~mm})$, filled with air and uranium. In the left panel, only POCAs with scattering angles $0<\theta<13 \mathrm{mrad}$ are included, whereas in the right panel only POCAs with scattering angles $\theta>13 \mathrm{mrad}$ are included.

by the fact that a large scattering angle indicates the presence of high- $Z$ material and also that the position accuracy of the POCA increases with larger scattering angles.

A typical result of the initial experiments is shown in Fig. 3. In the image the contrast, i.e., the signal to background ratio, is $15.0 \pm 0.8$. The image resolution, determined by fitting a WoodSaxon function to the edges of the reconstructed image was $2.1 \mathrm{~mm}$.

\subsection{Simulation of muon scattering}

Geant4 [13] has been used to simulate muon scattering in materials of significance for the long time storage of spent nuclear fuel, e.g., Fe and U. The emphasis was on determining the possibility of detecting the prescence of uranium in an iron environment and on determining whether it is possible to distinguish a single fuel bundle inside a canister for spent nuclear fuel or not.

In Fig. 4 it is shown how muon scattering allow voids, filled with air and uranium, inside an iron slab can be distinguished. By selecting POCAs with different scattering angles it is possible to put the emphasis of the selection towards lighter or heavier elements.

A model of a canister for spent nuclear fuel filled with typical fuel bundles, each containing $8 \times 8$ uranium fuel rods, was used to investigate the possibility of detecting if a single fuel bundle is missing as seen in Fig. 5. The resulting histogram was obtained by image subtraction. An image from a reference canister with all fuel bundles present was subtracted from an image of a canister with one fuel bundle missing and the result projected into a histogram. If the contents of the canisters are identical, only statistical fluctuations would remain. Position information from the POCAs has been used to put a geometrical gate that only selects events occuring in the third row of fuel bundle positions. The result was obtained from a flux of $6 \cdot 10^{5}$ muons, corresponding to a measurement time of 15 minutes. 

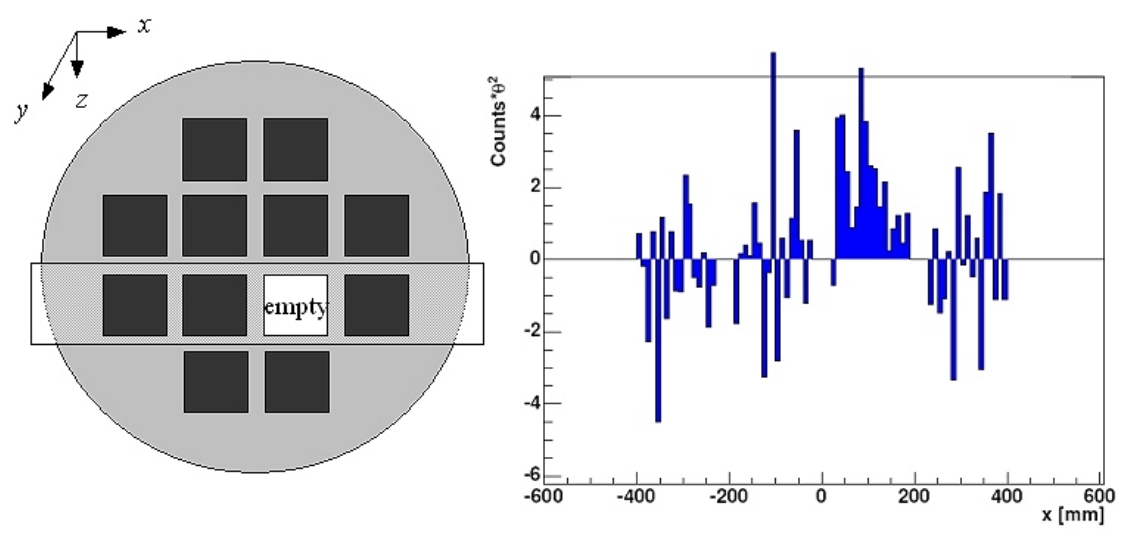

Figure 5: Simulation of a canister for spent nuclear fuel with one fuel bundle missing. The signal is obtained by image subtraction. A position gate, selecting POCAs within the rectangular area, is used to enhance the signal.

\section{Conclusion}

Fast neutron attenuation measurements and cosmic-ray muon scattering has potential for the imaging of canisters for spent nuclear fuel. High-energy neutron tomography offer the best possibility of high resolution imaging, albeit at high cost and with little possibility to distinguish between different materials. Cosmic-ray muon scattering tomography does not offer the high resolution of neutron tomography, but the method is much less expensive and offers some ability to distinguish between low, medium and high $Z$-materials. The method can be used to detect missing fuel bundles inside a sealed canister for spent nuclear fuel whithin a reasonable time span, i.e., 15 minutes. The POCA method for reconstruction of images is too simplistic for the intended application. More effort is needed in this area, as conventional tomographic transform-based methods cannot be used, but instead algebraic reconstruction methods must be utilised.

\section{Acknowledgments}

This work was financially supported by the Swedish Nuclear Power Inspectorate (SKI).

\section{References}

[1] Homepage of the Swedish Nuclear Fuel and Waste Management Co. (SKB), http://www.skb.se/, 2006.

[2] T.N. Taddeucci, C.L. Morris, K.B. Morley, J. Selden, http://lansce.lanl.gov, 1997.

[3] L.J. Schultz, et al., Nucl. Instr. and Meth. A 519, 687 (2004).

[4] R.W. Finlay, et al., Phys. Rev. C 47, 237 (1993).

[5] L.S. Waters, MCNPX User's Manual, Los Almos National Laboratory, September, 2002.

[6] L. Wallin, Uppsala University Neutron Physics Report, UU-NF 05\#02, February, 2005.

[7] J. Donnard, Rapport de synthèse, Uppsala University, Dept. of Neutron Res. Internal Report (2004).

[8] A. Flodin, Uppsala University Master of Science Thesis, UPTEC F05 035 (2005). 
[9] J. Gustafsson, Uppsala University Master of Science Thesis, UPTEC F05 077 (2005).

[10] L.W. Alvarez., Science 167, 832 (1970).

[11] K.N. Borozdin, et al., Nature 422, 277 (2003).

[12] D.E. Groom, N.V. Mokhov and S.I. Striganov, Atomic Data and Nuclear Data Tables 78, 183 (2001).

[13] S. Agostinelli, et al., Nucl. Instr. and Meth. A 506, 250 (2003). 